\title{
Maryna Viazovska to Receive the 2017 Sastra Ramanujan Prize
}

Krishnaswami Alladi (University of Florida, Gainesville, USA), Chair SASTRA Ramanujan Prize Committee

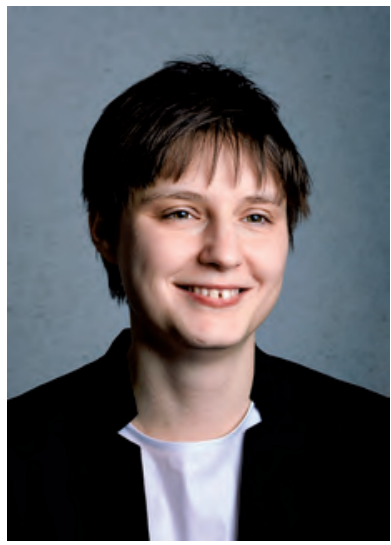

The 2017 SASTRA Ramanujan Prize will be awarded to Dr. Maryna Viazovska of the Swiss Federal Institute of Technology, Lausanne, Switzerland, especially for her stunning solution in dimension 8 of the celebrated sphere packing problem, and for her equally impressive joint work with Henry Cohn, Abhinav Kumar, Stephen D. Miller and Danylo Radchenko resolving the sphere packing problem in dimension 24 , by building upon her fundamental ideas in dimension 8.

The SASTRA Ramanujan Prize was established in 2005 and is awarded annually for outstanding contributions by young mathematicians to areas influenced by the genius Srinivasa Ramanujan. The age limit for the prize has been set at 32 because Ramanujan achieved so much in his brief life of 32 years. The prize will be awarded during December 21-22, 2017 at the International Conference on Number Theory at SASTRA University in Kumbakonam (Ramanujan's hometown) where the prize has been given annually.

Maryna Viazovska is an extraordinarily gifted mathematician who has made deep contributions to several fundamental problems in number theory. In her outstanding $\mathrm{PhD}$ thesis of 2013 written under the direction of Professor Don Zagier at the Max Planck Institute for Mathematics at the University of Bonn, Germany, she resolved the famous Gross-Zagier Conjecture in a substantial number of cases, including the important case pertaining to higher Green's functions that had been open for 30 years. Her thesis work was a tour-deforce making brilliant use of a variety of tools such as Borcherds lifts, Kudla's program on the arithmeticity of theta correspondences, and unusually clever technical calculations. This work of hers was published in the book Arithmetic and Geometry (Cambridge University Press, 2016).

Prior to joining the PhD program in Bonn, she had a number of impressive publications in collaboration with several active researchers. Particularly significant is her joint work with Andrii Bondarenko and Danylo Radchenko (a PhD student under Don Zagier in Bonn) which resolved a longstanding conjecture of Korevaar and Meyers on spherical designs, by giving an optimal upper bound for the minimal number of points in a spherical design. This work was published in the Annals of Mathematics (Princeton) in 2013. It is with this background that she started thinking about the sphere packing problem while in Bonn, the recent solution to which in dimensions 8 and 24 that propelled her into world prominence.

The sphere packing problem has a long and illustrious history. Johannes Kepler asked for the optimal way to assemble cannon balls (of uniform radius) and conjectured a configuration, but he could not prove it. This is the sphere packing problem in three dimensions, and can be generalized to arbitrary dimensions.

The sphere packing problem in three dimensions is known as Kepler's problem or The Kepler Conjecture. This conjecture in three dimensions was finally resolved by Thomas Hales in 1998 who gave a proof which was tour-de-force that combined ingenious geometric optimization arguments with machine calculations. The sphere packing problem in higher dimensions remained open. The sphere packing problem arises naturally not just in geometry and physics, but also in information theory, where sphere packings are error correcting codes for a continuous communication channel.

In dimension 8 there is $E_{8}$, an exceptional simple Lie group with a root lattice of rank 8 , and in dimension 24, we have the Leech Lattice, and both have remarkable structures. This gave some hope that the sphere packing problem could be resolved in dimensions 8 and 24 . Indeed Noam Elkies of Harvard University and Henry Cohn of Microsoft Research in Cambridge, Massachusetts, made significant progress by using the Poisson summation formula and linear programming bounds for the sphere packing density. They conjectured the existence of certain magic auxiliary functions in dimensions 8 and 24, which if determined, would resolve the conjecture in those dimensions. But these magic functions remained elusive. Viazovska produced these fuctions by an ingenious use of modular forms. Her attack was viewed as audacious, but when she succeeded, the mathematical world applauded in disbelief because her proof is remarkably simple. Paul Erdös, a legend of 20th century mathematics, has often joked that God has a Book containing the most beautiful proofs of the most important theorems; Viazovska's proof in dimension 8 is considered to be a proof from The Book! Her proof has been published in the Annals of Mathematics this year.

Once Viazovska had succeeded in dimension 8, the immediate question was whether her methods could be extended to dimension 24. Indeed, in the span of a week, by working at a furious pace, Viazovska in collaboration with Cohn, Kumar, Miller and Radchenko, successfully resolved the 24 dimensional case by building upon her 
ideas in dimension 8. This joint paper has also appeared this year in the Annals of Mathematics.

Viazovska's method makes crucial use of the theory of modular forms, which were a favorite of Ramanujan; indeed 24 is a number associated with Ramanujan's work. Viazovska's modular forms techniques are by no means limited to the sphere packing problem or ground states. She has discovered something profound that will play a broader role in discrete geometry, analytic number theory, and harmonic analysis.

The citation for the 2017 SASTRA Ramanujan Prize reads as follows:

"Maryna Viazovska is awarded the 2017 SASTRA Ramanujan Prize for her stunning and elegant resolution of the celebrated sphere packing problem in dimension 8, the proof of which appeared in her paper in the Annals of Mathematics (2017), and for her joint 2017 paper in the Annals of Mathematics with Henry Cohn, Abhinav Kumar, Stephen D. Miller and Danylo Radchenko, which resolves the sphere packing problem in dimension 24 by building on her ideas in dimension 8. The prize also recognizes her outstanding $P h D$ thesis of 2013 at the University of Bonn in which she resolved significant cases of the Gross-Zagier Conjecture and her work prior to her PhD with A. Bodarenko and $D$. Radchenko resolving a long standing conjecture of Korevaar and Meyers on spherical designs, that appeared in the Annals of Mathematics in 2013. The prize notes that the modular forms techniques developed by Viazovska will have a significant future impact in discrete geometry, analytic number theory, and harmonic analysis."

Maryna Viazovska, now 32 years old, was born in Kiev in the Ukraine on November 2, 1984. She completed her high school education in Kiev in 2001, and her BSc in Mathematics in 2005 at the Kiev National Taras Shevchenko University. She then went to Germany where she obtained a Masters degree in 2007 from the University of Kaiserslautern, after which she joined the University of Bonn that year. She graduate with a $\mathrm{PhD}$ from Bonn in 2013 writing a thesis under the direction of Professor Don Zagier. Since her PhD, she has received several awards and recognitions such as the Salem Prize in 2016 and the Clay Research Award in 2017. The SASTRA Ramanujan Prize is now a fitting recognition for her path-breaking work.

The 2017 SASTRA Ramanujan Prize Committee consisted of Professors

Krishnaswami Alladi - Chair (University of Florida),

Andrew Granville (University of Montreal)

Winfried Kohnen (University of Heidelberg)

Philippe Michel (EPF Lausanne)

Peter Sarnak (Princeton University and the Institute for Advanced Study)

Michael Schlosser (University of Vienna), and

Gisbert Wustholz (ETH, Zurich).
Previous winners of the Prize are

Manjul Bhargava and Kannan Soundararajan in 2005 (two full prizes)

Terence Tao in 2006

Ben Green in 2007

Akshay Venkatesh in 2008

Kathrin Bringmann in 2009

Wei Zhang in 2010

Roman Holowinsky in 2011

Zhiwei Yun in 2012

Peter Scholze in 2013

James Maynard in 2014

Jacob Tsimerman in 2015, and

Kaisa Matomaki and Maksym Radziwill (shared) in 2016.

The award of the 2017 SASTRA Ramanujan Prize to Maryna Viazovska is in keeping with the tradition of recognizing the spectacular contributions by the most brilliant young mathematicians.

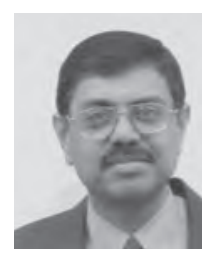

Krishnaswami Alladi is a professor of mathematics at the University of Florida where he was Department Chairman, 1998-2008. He received his $P h D$ from UCLA in 1978. His area of research is number theory. He is the founder and Editor-in-Chief of the Ramanujan Journal published by Springer. He helped create the SASTRA Ramanujan Prize and has chaired the prize committee since its inception. 